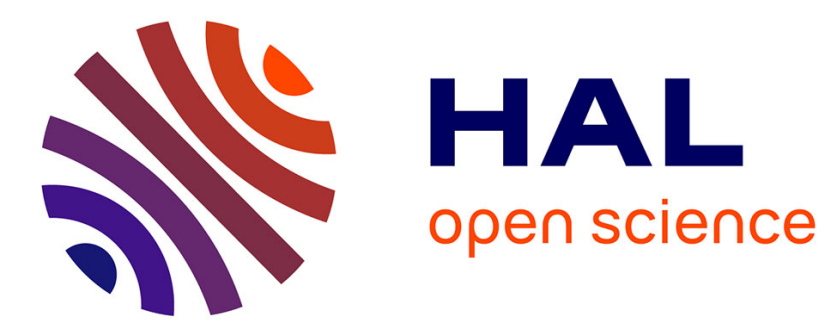

\title{
Cardiac Glycosides as Senolytic Compounds
}

Nadine Martin, Olivier Soriani, David Bernard

\section{To cite this version:}

Nadine Martin, Olivier Soriani, David Bernard. Cardiac Glycosides as Senolytic Compounds. Trends in Molecular Medicine, 2020, 26 (3), pp.243-245. 10.1016/j.molmed.2020.01.001 . hal-03009048

\section{HAL Id: hal-03009048 https://hal.science/hal-03009048}

Submitted on 17 Nov 2020

HAL is a multi-disciplinary open access archive for the deposit and dissemination of scientific research documents, whether they are published or not. The documents may come from teaching and research institutions in France or abroad, or from public or private research centers.
L'archive ouverte pluridisciplinaire HAL, est destinée au dépôt et à la diffusion de documents scientifiques de niveau recherche, publiés ou non, émanant des établissements d'enseignement et de recherche français ou étrangers, des laboratoires publics ou privés. 
2

4

5

6 Nadine MARTIN ${ }^{1, *}$, Olivier SORIANI ${ }^{2}$, David BERNARD ${ }^{1, *}$

7

$8 \quad{ }^{1}$ Centre de Recherche en Cancérologie de Lyon, Inserm U1052, CNRS UMR 5286, Université 9 de Lyon, Centre Léon Bérard, Lyon, France.

10

11

${ }^{2}$ Inserm, CNRS, iBV, Université Côte d’Azur, Nice, France.

12

13

14

nadine.martin@lyon.unicancer.fr

(N.Martin);

15 david.bernard@lyon.unicancer.fr (D.Bernard)

16

17

18

19

20 


\section{ABSTRACT}

22 The identification of senolytics, compounds able to eliminate senescent cells, is presently a key 23 challenge due to their therapeutic promises for cancer and aging-associated diseases. Two 24 recent papers by Triana-Martínez et al and Guerrero et al report the senolytic activity of cardiac 25 glycosides and their efficacy in these physiopathological contexts.

26

27

\section{KEYWORDS}

28

Cellular senescence, Senolytics, Cardiac glycosides, Cancer, Aging

29

30

31

32 
34 Cellular senescence can be induced by many stresses or during normal development. Upon senescence induction, cell cycle is stably arrested and cells secrete multiple proteins including pro-inflammatory cytokines and chemokines, growth factors and proteases. These two hallmarks of senescent cells are associated with changes in their metabolism, chromatin organization and gene expression. The senescence-associated secretory phenotype (SASP) reinforces senescence through autocrine activity and influences the microenvironment in a paracrine manner impacting tissue structure and function [1].

Cellular senescence can have a critical impact on the organism. It is mostly beneficial at a young age as it plays a key role in morphogenesis during normal embryonic development and it promotes tissue repair. Senescence is also beneficial, at least initially, in the context of protumoral stresses, as senescence activation is a barrier against tumorigenesis, by stopping the proliferation of pre-malignant cells and promoting their clearance by the immune system through the SASP [1].

However, during aging and/or upon exposure to chronic stress, persistent damage and defects in immune clearance lead to the accumulation of senescent cells which are expected to limit tissue renewal, create a chronic pro-inflammatory/pro-fibrotic microenvironment and impair tissue function. Recently, it was reported that accumulation of senescent cells promotes aging and many age-related disorders including cancer relapse and progression, cardiovascular diseases, obesity, type 2 diabetes, sarcopenia, lung fibrosis, osteoarthritis, atherosclerosis and neurological disorders. Indeed, genetically-engineered mouse models allowing the clearance of senescent cells show improved healthy aging, extended lifespan and delays or attenuation in many aging-associated pathologies during normal aging or after disease-inducing challenges [1]. 
These spectacular results prompted many laboratories and more recently biotechnology companies to search for compounds able to selectively kill senescent cells, named senolytics, to delay or improve age-related diseases. A potential difficulty to find such compounds is the intrinsic cell death resistance of senescent cells. Several potential senolytics have been identified in the last few years and some are already in clinical trials, mostly in a repositioning context. Others have notable side effects, preventing their use in the context of chronic diseases [2]. There is need for new senolytics for potential clinical translation as well as for improving our understanding of the mechanisms involved in the death resistance of senescent cells. Recently, publications by Triana-Martínez et al. [3] and Guerrero et al. [4] reported the identification of cardiac glycosides (CG) as a novel class of broad-spectrum senolytics after performing drug screens in models of oncogene-induced senescence in normal cells and therapy-induced senescence in normal and cancer cells, using libraries of mainly FDA/EMA approved drugs (Prestwick library), pharmacologically active compounds (LOPAC library) and natural compounds (GPNCL and SCREEN-WELL libraries).

The senolytic activity of CG was then assessed in vivo and ex vivo both in the context of cancer and aging-associated disorders. Ouabain, a natural compound obtained from the seeds of Strophanthus gratus and other plants of the Apocynaceae, killed senescent pre-neoplastic cells in a model of tumor initiation in liver and in adamantinomatous craniopharyngioma. In addition, in several models, digoxin, a CG obtained from the Digitalis plant and already used for treating heart failure and atrial fibrillation, and ouabain were highly efficient senolytics when used after senescence-inducing drugs (senogenics), a two-step therapeutic strategy proposed for tumour cells. Ouabain also diminished the number of bystander senescent cells which accumulate after irradiation and are responsible for some side effects. When administered in old mice, ouabain reduced the number of senescent cells, diminished the level of local inflammation and immune infiltration and improved some metabolic and physical fitness parameters that decline with 
aging. Digoxin, through its senolytic activity, also reduced lung fibrosis. Based on these findings, the authors proposed the potential use of CG as senolytics for therapeutic applications. Of note, digitoxin, a CG similar to digoxin, displays senolytic activity at concentrations similar to the ones measured in the plasma of patients treated with this compound for heart failure and atrial fibrillation. The description of the senolytic activity of CG also suggests they can act through this activity to exert previously described beneficial effects in the context of atherosclerosis, pulmonary fibrosis and anti-cancer treatment [5-7]. However, ouabain is able to induce premature senescence in immortalized human mammary epithelial cells [8], suggesting a cytostatic effect of CG in some proliferating cells and thus potential long-term deleterious effects.

CG are inhibitors of the $\mathrm{Na}^{+} / \mathrm{K}^{+}$ATPase, a plasma membrane (PM) enzyme pumping sodium out of and potassium into the cells against their respective concentration gradients and thereby regulating PM potential. Both publications provide data indicating that the senolytic activity of CG relies on the inhibition of the alpha subunit ATP1A1 of the $\mathrm{Na}^{+} / \mathrm{K}^{+}$ATPase. For example, inhibiting the $\mathrm{Na}^{+} / \mathrm{K}^{+}$ATPase by $\mathrm{KCl}$ treatment, since $\mathrm{K}+$ has a well-known antagonistic effect at CG binding sites [9], reverses the senolytic activity of CG.

Triana-Martínez et al further observed that senescent cells are slightly depolarized, which is in accordance with previous findings $[8,10,11]$, and that digoxin, by inhibiting the $\mathrm{Na}^{+} / \mathrm{K}^{+}$ ATPase, enhances PM depolarization of senescent cells. Triana-Martínez et al thus propose that enhancing PM depolarization contributes to the senolytic effect of CG. However, cell exposure to $\mathrm{KCl}$ is commonly used to trigger $\mathrm{PM}$ depolarization (as a direct consequence of the $\mathrm{K}^{+}$ equilibrium potential shift to more positive values according to Nernst's equation) and previous works showed that $\mathrm{KCl}$-induced depolarization by itself triggers cell senescence $[8,11]$, hampering the evaluation of the contribution of PM depolarization to the senolytic activity of CG. Triana-Martínez et al also suggest that the senolytic activity of CG might result from 
107 intracellular acidification, as increased $\mathrm{Na}^{+}$accumulation reduces $\mathrm{Na}^{+} / \mathrm{H}^{+}$exchanger activity, 108 resulting in intracellular $\mathrm{H}^{+}$accumulation.

109 Transcriptomic analysis performed by Guerrero et al revealed that ouabain and digoxin activate

110 the expression of genes of the pro-apoptotic Bcl-2 family. Among those, NOXA is induced by 111 the JNK, GSK3- $\beta$ and p38 pathways and partially mediates the senolytic effect of CG. How

112 CG lead to the activation of these pathways also remains to be determined.

113 In conclusion, the identification of CG as senolytics sheds light on new molecular players 114 regulating cellular senescence and new vulnerabilities of senescent cells. The two publications 115 describe the first pieces of a complex puzzle to explain how CG induce cell death. Signaling 116 pathways directly regulated by the $\mathrm{Na}^{+} / \mathrm{K}^{+}$ATPase, which involve SRC, ITPR and AKT among 117 others and are modulated by CG, could also play a role in the senolytic activity of these 118 compounds. Moreover, although the broad spectrum of action of CGs and their possible side 119 effects reduce the overall benefit of using these compounds for treatment, CG appear to be 120 promising candidates for therapeutic applications exploiting their senolytic effect in the context

121 of cancer and aging (Figure 1). A number of clinical trials with CGs are currently conducted, 122 especially combining CGs with chemotherapies for cancer treatment ${ }_{2}$ and novel ones will need 123 to be setup to assess their benefit against aging-related chronic diseases such as arthritis or 124 pulmonary fibrosis. 


\section{ACKNOWLEDGEMENTS}

127 We thank the Fondation ARC pour la recherche sur le cancer, the Institut National Contre le

128 Cancer (N²018-144), INSERM, Canceropole PACA, UCA ${ }^{\mathrm{JEDI}}$ and CNRS for their support.

129

\section{CONFLICT OF INTEREST}

131 None declared.

132

133

134 
136 1. He,S. and Sharpless,N.E. (2017) Senescence in Health and Disease. Cell 169, 1000-1011

137

138

139

140

141

142

143

144

145

146

147

148

149

150

151

152

153

154

155

156

157

158

159

160

161

162

2. Paez-Ribes,M. et al. (2019) Targeting senescent cells in translational medicine. EMBO Mol. Med. 11, e10234

3. Triana-Martinez,F. et al. (2019) Identification and characterization of Cardiac Glycosides as senolytic compounds. Nat. Commun. 10, 4731

4. Guerrero,A. et al. (2019) Cardiac glycosides are broad-spectrum senolytics. Nat. Metab 1, 1074-1088

5. Li,B. et al. (2018) Ouabain ameliorates bleomycin induced pulmonary fibrosis by inhibiting proliferation and promoting apoptosis of lung fibroblasts. Am. J. Transl. Res. 10, 2967-2974

6. Menger,L. et al. (2012) Cardiac glycosides exert anticancer effects by inducing immunogenic cell death. Sci. Transl. Med. 4, 143 ra99

7. Shi,H. et al. (2016) Digoxin reduces atherosclerosis in apolipoprotein E-deficient mice. Br. J. Pharmacol. 173, 1517-1528

8. Lallet-Daher,H. et al. (2013) Potassium Channel KCNA1 Modulates Oncogene-Induced Senescence and Transformation. Cancer Res. 73, 5253-5265

9. Laursen,M. et al. (2013) Crystal structure of the high-affinity Na+K+-ATPase-ouabain complex with Mg2+ bound in the cation binding site. Proc. Natl. Acad. Sci. U. S. A 110, 10958-10963

10. Carroll,B. et al. (2017) Persistent mTORC1 signaling in cell senescence results from defects in amino acid and growth factor sensing. J. Cell Biol. 216, 1949-1957

11. Warnier,M. et al. (2018) The SCN9A channel and plasma membrane depolarization promote cellular senescence through Rb pathway. Aging Cell 17, e12736 
164 Figure 1. Potential therapeutic applications of cardiac glycosides as senolytics. Selective

165 elimination of senescent cells (blue) by cardiac glycosides in different contexts (senescent

166 preneoplastic lesions, tumors treated with senescence-inducing therapies, lung fibrosis, old

167 organism) revealed several therapeutic benefits (green) for cancer, aging and its associated

168 diseases.

169

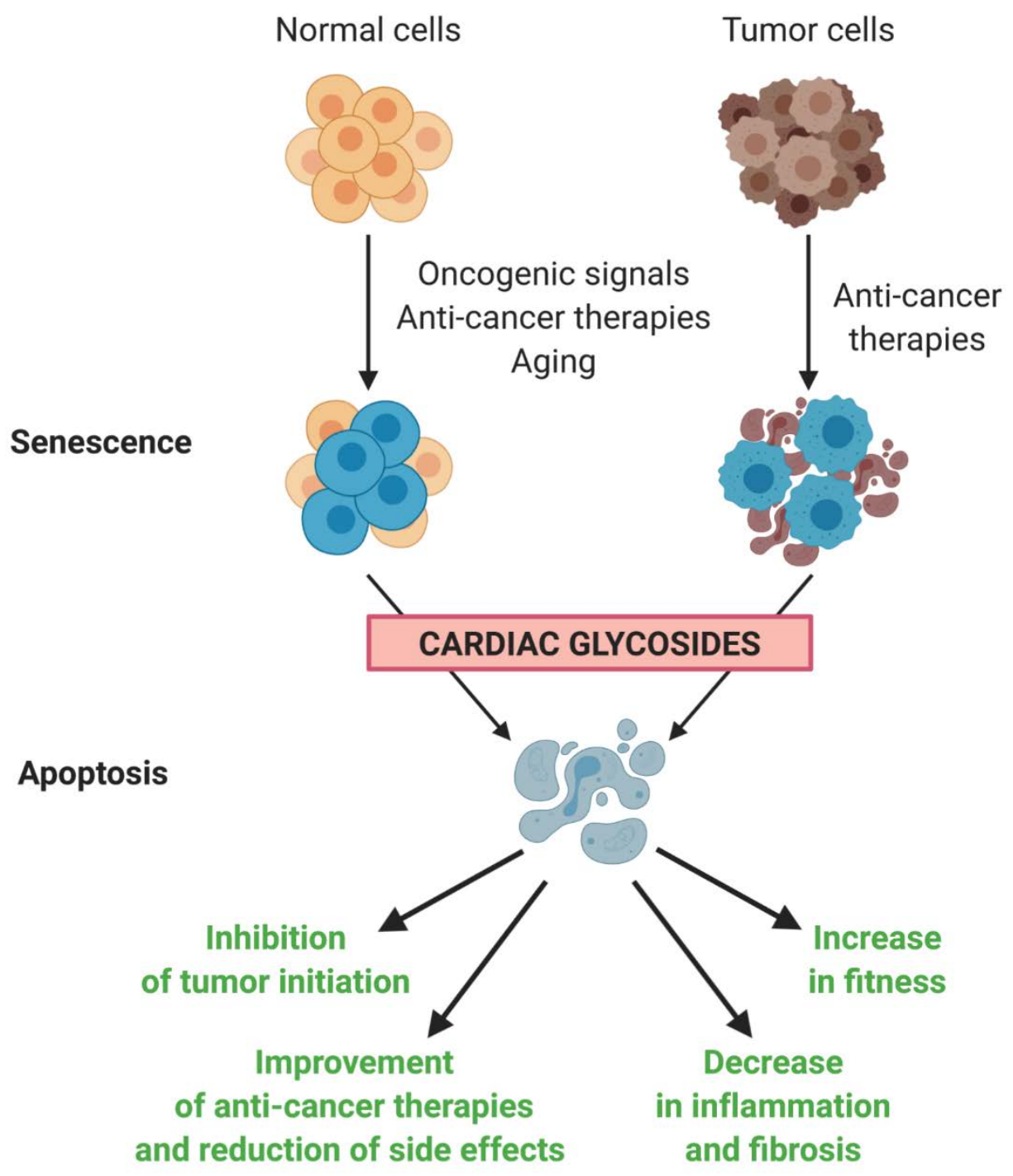

170 